\title{
Glaucoma, CTCAE
}

National Cancer Institute

\section{Source}

National Cancer Institute. Glaucoma, CT CAE. NCI Thesaurus. Code C55842.

A disorder characterized by an increase in pressure in the eyeball due to obstruction of the aqueous humor outflow. 